\title{
Identification of key N6-Methyladenosine-Related IncRNAs in Colon Adenocarcinoma using Bioinformatics Analysis
}

\section{Yuancheng Huang}

Guangzhou University of Chinese Medicine

\section{Yanhua Yan}

Guangzhou University of Chinese Medicine

Chaoyuan Huang

Guangzhou University of Chinese Medicine

\section{Xiaotao Jiang}

Guangzhou University of Chinese Medicine

\section{Zehong Yang}

Guangzhou University of Chinese Medicine

Kunhai Zhuang

Guangzhou University of Chinese Medicine

Fengbin Liu

Guangzhou University of Chinese Medicine

\section{Peiwu Li}

Guangzhou University of Chinese Medicine

Yi Wen ( $\nabla$ doctorwenyigzucm@163.com )

Guangzhou University of Chinese Medicine

\section{Research Article}

Keywords: colon adenocarcinoma, N6-methyladenosine (m6A), long non-coding RNAs (IncRNAs), prognostic signature, TCGA.

Posted Date: July 28th, 2021

DOI: https://doi.org/10.21203/rs.3.rs-734870/v1

License: (c) (1) This work is licensed under a Creative Commons Attribution 4.0 International License. Read Full License 


\section{Abstract}

Purpose: The purpose of this study was to investigate the role of $\mathrm{m}^{6} \mathrm{~A}$-related IncRNAs in colon adenocarcinoma (COAD) and determine their prognostic value.

Material and methods: Gene expression and clinicopathological data were obtained from The Cancer Genome Atlas database. Correlation and univariate Cox regression analysis were conducted to identify $\mathrm{m}^{6}$ A-related prognostic IncRNAs. A prognostic signature was established via least absolute shrinkage and selection operator (LASSO) Cox regression analyses. The prognostic value of risk scores was evaluated using the Kaplan-Meier method, receiver operating characteristic curves, and univariate and multivariate regression analyses. Whether the prognostic model could serve as a prognostic indicator for overall survival (OS) in subgroups of patients with different clinical characteristics were explored. Next, We established a competing endogenous RNA network. Gene Set Enrichment Analysis, Kyoto Encyclopedia of Genes and Genomes pathway, and Gene Ontology analysis were performed for biological functional analysis.

Results: 36 IncRNAs that were highly correlated with OS of patients were identified. A prognostic signature comprising $11 \mathrm{~m}^{6} \mathrm{~A}$-related IncRNAs was constructed, which had significant value in predicting the OS of patients. Univariate and Multivariate Cox regression analyses suggested that the risk score was an independent prognostic factor. This $\mathrm{m}^{6} \mathrm{~A}$-related IncRNA prognostic model could serve as a prognostic indicator for OS in subgroups of patients with different clinical characteristics. Biological processes and pathways associated with cancer were identified.

Conclusion: We revealed the role and prognostic value of $\mathrm{m}^{6} \mathrm{~A}$-related IncRNAs in COAD. Our finding refreshed the understanding of $\mathrm{m}^{6} \mathrm{~A}$-related IncRNAs and provided novel insights to identify predictive biomarkers and develop targeted therapy for COAD.

\section{Introduction}

Globally, colorectal cancer (CRC) is the third most common cancer and the second most deadly neoplasm ${ }^{1}$. Colon adenocarcinoma (COAD) is the most common pathological type of CRC, and despite considerable progress in diagnosis and therapeutic strategies for COAD, the prognosis of patients with COAD remains poor due to advanced stage and postsurgical recurrence ${ }^{2,3}$. Therefore, identification of novel biomarkers for early detection and effective therapeutic targets for treating patients with COAD is critical and urgent.

Accumulating evidence has shown that long non-coding RNAs (IncRNAs) had various biological functions and played a crucial role in the oncogenesis and progression of $\mathrm{CRC}^{4}$. For example, IncRNA ACTA2-AS1 functions as a competing endogenous RNA (ceRNA) to miR-4428 and promotes the pathogenicity of COAD by regulating BCL2L11 ${ }^{5}$. LncRNA MNX1-AS1 acts as an oncogene in CRC by 
interacting with RNA binding protein $\mathrm{YB} 1^{6}$. LINC00337 could facilitate the tumorigenesis and angiogenesis in CRC via recruiting DNMT1 to restrict the expression of CNN1 ${ }^{7}$.

Increasing evidence suggests that RNA modifications play a critical roles in tumorigenesis and progression of different cancers, including $C R C^{8,9}$. N6-methyladenosine $\left(\mathrm{m}^{6} \mathrm{~A}\right)$, introducing a methyl group in the nitrogen- 6 position of adenosine, is found to be the most frequent internal RNA modification in mammals ${ }^{10}$. As a dynamic and reversible process, $m^{6} \mathrm{~A}$ RNA modification is primarily regulated by "writers" (adenosine methyltransferases) and "erasers" (demethylases), and performs different functions by interacting with "readers" ( ${ }^{6} \mathrm{~A}$-binding proteins). As identified to distribute extensively in a variety of RNAs, such as messenger RNAs (mRNAs), pri-microRNAs (pri-miRNAs), circular RNAs (circRNAs) and IncRNAs, $\mathrm{m}^{6} \mathrm{~A}$ is involved in various biological processes related to the occurrence and progression of tumors, including $\mathrm{CRC}^{11}$. For instance, the $\mathrm{m}^{6} \mathrm{~A}$ writer METTL3 stimulates $\mathrm{m}^{6} \mathrm{~A}$ modification of CCNE1 mRNA and enhances its stability, which CRC proliferation ${ }^{12}$. Overexpression METTL3 facilitates processing of pri-miR-1246 into the miR-1246 through an $\mathrm{m}^{6} \mathrm{~A}$ DGCR8-dependent method, which activates the MAPK pathway and the progression of $C R C^{13}$. YTHDF3 recognizes and binds to $m^{6} A-$ modified IncRNA GAS5 and promotes its degradation, which elevates YAP expression and promotes $\mathrm{CRC}^{14}$.

Here, we analyzed the Cancer Genome Atlas (TCGA) database for $\mathrm{m}^{6}$ A-related IncRNAs involved in COAD and established a ${ }^{6}$ A-related IncRNAs prognostic signature. Then, we demonstrated that the $\mathrm{m}^{6} \mathrm{~A}$ related IncRNA prognostic model could serve as a prognostic indicator for overall survival (OS) in subgroups of patients with different clinical characteristics. Furthermore, a ceRNA network was constructed based on the $\mathrm{m}^{6} \mathrm{~A}$-related prognostic IncRNAs in COAD. The finding in this study revealed the critical role of $\mathrm{m}^{6} \mathrm{~A}$-related IncRNAs and shed light on the latent relationship and underlying mechanism between $\mathrm{m}^{6} \mathrm{~A}$-related IncRNAs and COAD.

\section{Material And Methods}

\section{Acquisition of datasets}

The RNA-seq transcriptome data (fragments per kilobase million, FPKM) ${ }^{15}$ from 437 samples and clinical information from 385 patients with COAD in TCGA database (http://cancergenome.nih.gov/) were downloaded for our study. Patients with complete clinicopathological and survival information were included for further assessment.

\section{Selection of $\mathrm{m}^{6} \mathrm{~A}$-related regulators}

Based on published data ${ }^{8}, 24 \mathrm{~m}^{6} \mathrm{~A}$-related regulators, including METTL3, METTL14, METTL16, WTAP, VIRMA, KIAA1429, ZC3H13 RBM15, RBM15B, YTHDC1, YTHDC2, YTHDF1, YTHDF2, YTHDF3, HNRNPC, FMR1, LRPPRC, HNRNPA2B1, IGFBP1, IGFBP2, IGFBP3, RBMX, FTO and ALKBH5, were used in our study. 


\section{Bioinformatic Analysis}

Primarily, correlation analysis was performed between $\mathrm{m}^{6} \mathrm{~A}$-related regulators and all IncRNAs in COAD. $\mathrm{m}^{6} \mathrm{~A}$-related IncRNAs were identified based on the following classification parameters: 1 ) correlation coefficients more than 0.3 ; 2$) p$-value less than 0.001 . Then, to filtrate the $\mathrm{m}^{6} \mathrm{~A}$-related IncRNAs that were highly correlated with OS, univariate Cox regression analysis was performed. Next, we randomly divided the patients with COAD into two groups: the training group and the testing group. Subsequently, based on $\mathrm{m}^{6} \mathrm{~A}$-related prognostic IncRNAs identified by univariate Cox regression analysis, the least absolute shrinkage and selection operator (LASSO) Cox regression algorithm was used to identify $\mathrm{m}^{6} \mathrm{~A}$-related IncRNAs with powerful prognostic significance and construct the prognostic risk model from the training group data. According to the best penalty parameter $\lambda$, the $\mathrm{m}^{6}$ A-related IncRNAs' coefficients were calculated. The risk score (RS) was estimated using the following formula:

$$
R S=\sum_{i=1}^{\mathrm{n}} \operatorname{Coef}(i) X(i)
$$

where Coef(i) is the coefficient and X(i) represents the expression levels of $m^{6} A$-related IncRNAs. Using the obtained median RS as the demarcation value, patients with COAD were classified in two groups: high-risk group and low-risk group. Kaplan-Meier analysis and the receiver operating characteristic (ROC) curves were used to validate the predictive efficiency ${ }^{16}$. Then, the accuracy of the model was validated from the test group and the combined group by the same method. Furthermore, the differences in clinicopathological features between high-risk group and low-risk group were also explored. Additionally, the prognostic value of the RS was verified using univariate and multivariate Cox regression analyses. The hazard ratio (HR) with 95\% confidence intervals and log-rank $p$-value were calculated using the "glmnet" and "survival" R packages ${ }^{17}$.

To explore the biological functions associated with $\mathrm{m}^{6} \mathrm{~A}$-related IncRNAs, Gene Set Enrichment Analysis (GSEA), Kyoto Encyclopedia of Genes and Genomes (KEGG) pathway and Gene Ontology (GO) analysis were performed. Genes in different risk groups were functionally annotated using GSEA. Based on the $\mathrm{m}^{6} \mathrm{~A}$-related prognostic IncRNAs, the target miRNAs were predicted via miRcode database and target mRNAs of these miRNAs were found in different databases, such as TargetScan, miRTarBase, and miRDB. Target mRNAs in the ceRNA network were functionally annotated using GO and KEGG pathway analysis. The flow chart of bioinformatic analysis was shown in Fig. 1.

\section{Statistical analysis}

The expression data of $\mathrm{m}^{6} \mathrm{~A}$-related regulators and all IncRNAs in tumor tissues and adjacent mucosa of COAD obtained from TCGA was compared using one-way analysis of variance (ANOVA); the clinical characteristics of different groups were compared using the chi-square test; the Kaplan-Meier method was used to perform a bilateral logarithmic rank test in OS analysis; $p$-values $<0.05$ were regarded as 
statistically significant. All statistical analyses were implemented using R v4.0.3 (https://www.rproject.org/).

\section{Results}

\section{Identification of $\mathrm{m}^{6} \mathrm{~A}$-Related prognostic IncRNAs}

Firstly, the expression levels of $24 \mathrm{~m}^{6} \mathrm{~A}$-related genes and all IncRNAs from the TCGA were extracted respectively. Through coexpression analysis, we identified $581 \mathrm{~m}^{6} \mathrm{~A}$-related $\operatorname{lncRNAs}(|\operatorname{cor}|>0.3, p$-value $<$ 0.001). The gene co-expression network of $24 \mathrm{~m}^{6} \mathrm{~A}$-related genes and $581 \mathrm{~m}^{6} \mathrm{~A}$-related IncRNAs was shown in Fig. 2A. After conducting univariate Cox analysis, 36 candidate IncRNAs that were highly correlated with OS were identified ( $p<0.05)$ (Fig. 2B). The expression of $36 \mathrm{~m}^{6} \mathrm{~A}$-Related prognostic IncRNAs between tumor tissues and adjacent mucosa was compared (Fig. 2C, D). Among these IncRNAs, two IncRNAs (LINC01555, SNHG16) were prognostic protective factors and others were prognostic risk factors.

\section{Construction and Verification of the $\mathrm{m}^{6} \mathrm{~A}-$ Related IncRNAs Prognostic Signature}

Based on 36 candidate IncRNAs that were highly correlated with OS, we used the LASSO method in the training group to construct a $\mathrm{m}^{6} \mathrm{~A}$-related IncRNAs signature for evaluating the prognosis of patients with COAD. Finally, 11 IncRNAs were chosen to establish a prognostic signature and the risk score was calculated (Fig. 3A, B). Using the median risk score as the demarcation value, patients in the training group $(n=190)$ were classified into two groups, namely the high-risk group and low-risk group. To test the efficacy of the prognostic model, survival and ROC curves analyses were conducted. Kaplan-Meier analysis showed that the low-risk group had significantly longer survival time than the high-risk group ( $p$ $<0.001$ ) (Fig. 3C). The area under the curve (AUC) value in the time-dependent ROC curve of 1-, 3- and 5year was $0.768,0.814$ and 0.873 severally(Fig. 3D), suggesting good prediction performance of the survival model. To further validate this 11 IncRNAs prognostic signature, verification analysis in the test group ( $n=189)$ and the combined group $(n=379)$ were implemented. As a result, the high-risk group in the test group and the combined group had significantly shorter survival time compared with the low-risk group, which was previously observed in the training group (Fig. 3C). Time-dependent ROC curve of the test group and the combined group also had well-prediction performances, and the AUC value of 1-,3- and 5 -year was shown in Fig. 3D. The risk scores, survival time and the expression of $11 \mathrm{~m}^{6} \mathrm{~A}$-related prognostic IncRNAs of the high-risk group and low-risk group in different subgroups were displayed in Fig. 3E, F, G).

To examine whether the risk score was an independent prognostic factor, univariate and multivariate Cox regression analyses were conducted. This revealed that the risk score $(p<0.001)$ was significantly 
associated with OS in patients with COAD, in addition to age at diagnosis $(p<0.05)$ and pathological stage $(p<0.001)$ (Fig. 4A, B).

\section{Subgroup Analysis with Different Clinicopathological Features}

The expression of $11 \mathrm{~m}^{6} \mathrm{~A}$-related prognostic IncRNAs and the distribution of clinicopathological characteristics in the high-risk group and low-risk group were displayed as a heatmap (Fig. 4C). Evident differences between the two groups according to pathological stage $(p<0.001)$, T stage $(p<0.001)$, M stage $(p<0.001)$ and N stage $(p<0.001)$ were observed. Significant differences of risk score were found between: 1) different pathological stage $(p<0.001) ; 2)$ different T stage $(p<0.001) ; 3)$ different M stage $(p<0.001)$; and 3) different $\mathrm{N}$ stage $(p<0.001)$ (Fig. 4D).

To evaluate whether $\mathrm{m}^{6} \mathrm{~A}$-related IncRNA prognostic model could serve as a prognostic indicator for OS in subgroups of patients with different clinical characteristics, we stratified subgroups by age (age $\leq 65$ and age $>65$ ), gender (female and male), clinical stage (stage I-II and stage III-IV), T stage (T1-2 and T3-4), M stage (M0 and M1) and N stage (N0 and N1-3). As the result shown in Fig. 5, the OS of the low-risk patients based on age $(p<0.001)$, sex $(p=0.001$ in female and $p<0.001$ in male), pathological stage ( $p=$ 0.002 in stage I-II and $p=0.004$ in stage III-IV), T stage ( $p=0.014$ in T1-2 stage and $p<0.001$ in T3-4 stage), M0 stage $(p<0.001)$ and $\mathrm{N}$ stage $(p<0.001$ in $\mathrm{N} 0$ and $=0.006$ in N1-3) were significantly higher than those of the high-risk patients.

\section{Construction of the ceRNA Network and Functional Enrichment Analysis}

To explore the biological function of $36 \mathrm{~m}^{6} \mathrm{~A}$-related prognostic IncRNAs, a ceRNA network was constructed based on the mechanism of IncRNAs regulating mRNA expression by sponging miRNAs. 5 IncRNAs were extracted from the miRcode database and 28 pairs of interaction between the 5 IncRNAs and 31 miRNAs were identified. Based on three mRNA predicting database mentioned previously and differentially expressed mRNA between normal group and tumor group of COAD in TCGA, we totally identified 216 target mRNA. Finally, 5 IncRNAs, 31 miRNAs and 216 mRNAs were included to construct ceRNA by Cytoscape software 3.7.1 (Fig. 6A). KEGG pathway and GO analysis were performed to annotate the function of 216 target mRNAs and we found that these target mRNAs were enriched in DNAbinding transcription activator activity, and DNA-binding transcription repressor activity (GO analysis) (Fig. 6B); MicroRNAs in cancer, PI3K-Akt signaling pathway, MAPK signaling pathway, p53 signaling pathway, Cell cycle and Focal adhesion (KEGG pathways) (Fig. 6C).

Furthermore, we used GSEA to predict the functional difference between high-risk group and low-risk group. The results showed that high-risk group was closely enriched in the cancer-related pathways, such as “Focal adhesion," “ECM-receptor interaction,” and "Wnt signaling pathway."(Fig. 6D) 


\section{Discussion}

Recently, an increasing number of studies focusing on the role of IncRNAs in CRC proved that IncRNAs exert a critical oncogenic role based on its dysregulated expression and localization ${ }^{18,19}$. Additionally, as the most abundant posttranscriptional modification in eukaryotic non-coding RNAs (ncRNAs), ${ }^{6} \mathrm{~A}$ has a huge effect on its stability and transport ${ }^{20-22}$. Previous studies have shown that $\mathrm{m}^{6} \mathrm{~A}$ "writers" and "erasers" could adjust the levels of $\mathrm{m}^{6} \mathrm{~A}$ modification in mRNAs and ncRNAs to regulate binding sites to $\mathrm{m}^{6} \mathrm{~A}$ "reader" proteins. Different $\mathrm{m}^{6} \mathrm{~A}$ "reader" proteins recognize and bind to methylated ncRNAs to realize different functions. For instance, the $\mathrm{m}^{6} \mathrm{~A}$ mark increases the stability of IncRNA FAM225A, which promotes nasopharyngeal carcinoma progression by acting as ceRNA to sponge miR-590-3p/miR$1275^{23}$. IGF2BP2 recognizes and binds to $\mathrm{m}^{6} \mathrm{~A}$-modified circRNA NSUN2 and increases its export to the cytoplasm ${ }^{24}$. Overexpression METTL3 can significantly increase IncRNA RP11 nuclear localization in $\mathrm{CRC}$ cells ${ }^{25}$. Thus, in consideration of the crucial role of IncRNAs and $\mathrm{m}^{6} \mathrm{~A}$ RNA modification in COAD, these researches call our attention to investigate the gene profile of $\mathrm{m}^{6} \mathrm{~A}$-related IncRNAs and molecular mechanisms involved in COAD, explore whether $\mathrm{m}^{6} \mathrm{~A}$-related IncRNAs could serve as ideal biomarkers for COAD prognosis and participate in COAD initiation and progression.

In our study, a total of 437 samples, 385 patients with COAD, $24 \mathrm{~m}^{6} \mathrm{~A}$-related regulators and 3910 IncRNAs were included to exploit the specific role of $\mathrm{m}^{6} \mathrm{~A}$-related IncRNAs in COAD. 36 candidate IncRNAs that were highly correlated with OS of COAD were identified. Then, 11 of $36 \mathrm{~m}^{6} \mathrm{~A}$-related prognostic IncRNAs were used to establish a prognostic signature with the LASSO method in the training group. Kaplan-Meier analysis showed that the OS of patients with low risk scores was longer than those of patients with high risk scores. Additionally, the result of ROC curve analysis indicated that the 11-IncRNAs signature could serve as a highly specific and sensitive prognostic survival model in COAD. Moreover, the results were further validated in the test groups and the combined group. This signature can be used as an independent prognostic factor for COAD, suggesting that these 11 IncRNAs may be vital $\mathrm{m}^{6} \mathrm{~A}$-related IncRNAs and significant prognostic factors for patients with COAD. Furthermore, this $\mathrm{m}^{6} \mathrm{~A}$-related IncRNA prognostic model could serve as a prognostic indicator for OS in subgroups of patients with different clinical characteristics, especially age, gender, pathological stage, T stage, M0 stage and $\mathrm{N}$ stage.

To provide a comprehensive analysis of $\mathrm{m}^{6} \mathrm{~A}$-related IncRNAs, a ceRNA network consisting of 5 IncRNAs, 31 miRNAs and 216 mRNAs was constructed and differentially expressed genes between the high-risk group and low-risk group were identified for viewing the latent functions of $\mathrm{m}^{6} \mathrm{~A}$-related IncRNAs. With the 5 key $\mathrm{m}^{6} \mathrm{~A}$-related IncRNAs, LINC00174 and ZEB1-AS1 have been preliminarily studied so far in COAD. LncRNA ZEB1-AS1 acts as a sponge of miR-141-3p/miR-205/miR-455-3p/miR-181a-5p/miR-101 to promote COAD malignant progression ${ }^{25}$. LINC00174 was overexpressed in CRC tissues and cells, and promotes CRC progression via maintaining TAZ overexpression by sponging miR-1910-3p ${ }^{31}$. KEGG pathway and GO analysis showed that 216 target mRNAs were enriched in several biological processes and pathways associated with the occurrence and progression of $\mathrm{COAD}^{32,33}$, including "PI3K-Akt 
signaling pathway," "MAPK signaling pathway," "p53 signaling pathway," "Cell cycle," "Focal adhesion” and so on. Genes in high-risk group and low-risk group were functionally annotated using GSEA. Genes in high-risk group were also enriched in cancer-related pathway,such as "Focal adhesion," "ECM-receptor interaction," and "Wnt signaling pathway."

However, a few limitations and shortcomings in our study should be acknowledged. First, it's beneficial to perform external validation by other genes and clinical datasets. Second, the results of this study is purely computational, and further experimental studies are necessary.

\section{Conclusion}

In this study, we constructed a prognostic signature comprising $11 \mathrm{~m}^{6}$ A-related IncRNAs in COAD, which had significant value in predicting the OS of patients with COAD. Additionally, biological processes and pathways associated with $\mathrm{m}^{6} \mathrm{~A}$-related IncRNAs were identified, which improved our understanding of the role of $\mathrm{m}^{6} \mathrm{~A}$-related IncRNAs in the occurrence and progression of COAD. This work also provides important evidence towards the development of predictive biomarkers and molecular targeted therapy for COAD.

\section{Declarations}

\section{Availability of Data and Materials}

Publicly available datasets were analyzed in this study, which can be found in the Cancer Genome Atlas (TCGA) database.

\section{Ethics Approval and Consent to Participate}

Not applicable

\section{Consent for Publication}

All the authors consented to the publication of this research.

\section{Competing Interests}

The authors declare that the research was conducted in the absence of any commercial or financial relationships that could be construed as a potential conflict of interest.

\section{Author Contributions}

$\mathrm{YH}, \mathrm{PL}, \mathrm{FL}$ and $\mathrm{YW}$ conceived and designed the study. $\mathrm{YH}, \mathrm{YY}, \mathrm{CH}, \mathrm{XJ}$, and $\mathrm{ZY}$ organized the database and performed statistical analyses. $\mathrm{YH}$ and $\mathrm{YW}$ wrote the first draft of the manuscript. $\mathrm{YY}, \mathrm{CH}$ and $\mathrm{XJ}$ prepared the figures and tables and were involved in manuscript writing. KZ, FL, PL and YW revised and proofread the manuscript. All authors contributed to manuscript revision and approved the submitted version. 


\section{Funding}

This study was supported by The National Natural Science Foundation (No. 81904139, No. 81973819 and No. 81904145), Collaborative Innovation Team Project of First-Rate Universities and Disciplines and High-level University Discipline of Guangzhou University of Chinese Medicine (No. 2021xk47), The Natural Science Foundation of Guangdong Province (No. 2019A1515011145), Guangdong Medical Science and Technology Research Fund (No. B2021089, No. A2020186), Clinical Research Project of Innovation Hospital in the First Affiliated Hospital of Guangzhou University of Chinese Medicine (No. 2019هT19) and High-level Hospital Construction project of the First Affiliated Hospital of Guangzhou University of Chinese Medicine (No. 2019QN01).

\section{Acknowledgments}

This study benefited from the Cancer Genome Atlas (TCGA) databases. We appreciate the data platform and the authors up-loaded their data.

\section{Disclosure of Potential Conflicts of Interest}

The author reports no conflicts of interest in this work.

\section{Research involving Human Participants and/or Animals}

Not applicable

\section{Informed Consent}

Not applicable

\section{References}

1. Miller KD, Nogueira L, Mariotto AB, Rowland JH, Yabroff KR, Alfano CM, Jemal A, Kramer JL, Siegel RL (2019) Cancer treatment and survivorship statistics, 2019. CA Cancer J Clin 69:363-385. doi:10.3322/caac. 21565

2. Bray F, Ferlay J, Soerjomataram I, Siegel RL, Torre LA, Jemal A (2018) Global cancer statistics 2018: GLOBOCAN estimates of incidence and mortality worldwide for 36 cancers in 185 countries. CA Cancer J Clin 68:394-424. doi:10.3322/caac.21492

3. White A, Joseph D, Rim SH, Johnson CJ, Coleman MP, Allemani C (2017) Colon cancer survival in the United States by race and stage (2001-2009): Findings from the CONCORD-2 study. CANCER-AM CANCER SOC 123(Suppl 24):5014-5036. doi:10.1002/cncr.31076

4. Schwarzmueller L, Bril O, Vermeulen L, Léveillé N. Emerging Role and Therapeutic Potential of IncRNAs in Colorectal Cancer. Cancers (Basel).(2020)12. doi:10.3390/cancers12123843 
5. Pan Q, Huang Y, Wang Y, Li D, Lei C (2021) LncRNA ACTA2-AS1 suppress colon adenocarcinoma progression by sponging miR-4428 upregulation BCL2L11. CANCER CELL INT 21:203. doi:10.1186/s12935-021-01769-3

6. Wu QN, Luo XJ, Liu J, Lu YX, Wang Y, Qi J, Liu ZX, Huang QT, Liu ZK, Lu JB, Jin Y, Pu HY, Hu PS, Zheng JB, Zeng ZL, Ju HQ, Xie D, Zhao Q, Xu R (2021) MYC-Activated LncRNA MNX1-AS1 Promotes the Progression of Colorectal Cancer by Stabilizing YB1. CANCER RES. doi:10.1158/0008-5472.CAN20-3747

7. Xu X, Nie J, Lu L, Du C, Meng F, Song D (2020) LINC00337 promotes tumor angiogenesis in colorectal cancer by recruiting DNMT1, which suppresses the expression of CNN1. CANCER GENE THER. doi:10.1038/s41417-020-00277-2

8. Jiang X, Liu B, Nie Z, Duan L, Xiong Q, Jin Z, Yang C, Chen Y (2021) The role of m6A modification in the biological functions and diseases. Signal transduction targeted therapy 6:74-74. doi:10.1038/s41392-020-00450-x

9. Xie S, Chen W, Chen K, Chang Y, Yang F, Lin A, Shu Q, Zhou T, Yan X (2020) Emerging roles of RNA methylation in gastrointestinal cancers. CANCER CELL INT 20:585. doi:10.1186/s12935-020-01679w

10. Nombela P, Miguel-López B, Blanco $S$ (2021) The role of $m(6) A, m(5) C$ and $\Psi$ RNA modifications in cancer: Novel therapeutic opportunities. MOL CANCER 20:18. doi:10.1186/s12943-020-01263-w

11. Fang Z, Hu Y, Hu J, Huang Y, Zheng S, Guo C (2021) The crucial roles of N(6)-methyladenosine $(m(6) A)$ modification in the carcinogenesis and progression of colorectal cancer. CELL BIOSCI 11:72. doi:10.1186/s13578-021-00583-8

12. Zhu W, Si Y, Xu J, Lin Y, Wang JZ, Cao M, Sun S, Ding Q, Zhu L, Wei JF (2020) Methyltransferase like 3 promotes colorectal cancer proliferation by stabilizing CCNE1 mRNA in an m6A-dependent manner. J CELL MOL MED 24:3521-3533. doi:10.1111/jcmm.15042

13. Peng W, Li J, Chen R, Gu Q, Yang P, Qian W, Ji D, Wang Q, Zhang Z, Tang J, Sun Y (2019) Upregulated METTL3 promotes metastasis of colorectal Cancer via miR-1246/SPRED2/MAPK signaling pathway. J Exp Clin Cancer Res 38:393. doi:10.1186/s13046-019-1408-4

14. Ni W, Yao S, Zhou Y, Liu Y, Huang P, Zhou A, Liu J, Che L, Li J (2019) Long noncoding RNA GAS5 inhibits progression of colorectal cancer by interacting with and triggering YAP phosphorylation and degradation and is negatively regulated by the m(6)A reader YTHDF3. MOL CANCER 18:143. doi:10.1186/s12943-019-1079-y

15. Mortazavi A, Williams BA, McCue K, Schaeffer L, Wold B (2008) Mapping and quantifying mammalian transcriptomes by RNA-SEq. NAT METHODS 5:621-628. doi:10.1038/nmeth.1226

16. Hanley JA, McNeil BJ. The meaning and use of the area under a receiver operating characteristic (ROC) curve. RADIOLOGY.(1982)143: 29-36. doi:10.1148/radiology.143.1.7063747

17. Simon N, Friedman J, Hastie T, Tibshirani R (2011) Regularization Paths for Cox's Proportional Hazards Model via Coordinate Descent. J STAT SOFTW 39:1-13. doi:10.18637/jss.v039.i05 
18. Chen L, He M, Zhang M, Sun Q, Zeng S, Zhao H, Yang H, Liu M, Ren S, Meng X, Xu H (2021) The Role of non-coding RNAs in colorectal cancer, with a focus on its autophagy. Pharmacol Ther 226:107868. doi:10.1016/j.pharmthera.2021.107868

19. Wang L, Cho KB, Li Y, Tao G, Xie Z, Guo B. Long Noncoding RNA (IncRNA)-Mediated Competing Endogenous RNA Networks Provide Novel Potential Biomarkers and Therapeutic Targets for Colorectal Cancer. INT J MOL SCl.(2019)20. doi:10.3390/ijms20225758

20. He RZ, Jiang J, Luo DX (2020) The functions of N6-methyladenosine modification in IncRNAs. Genes Dis 7:598-605. doi:10.1016/j.gendis.2020.03.005

21. Zhang L, Hou C, Chen C, Guo Y, Yuan W, Yin D, Liu J, Sun Z (2020) The role of N(6)-methyladenosine $(\mathrm{m}(6) \mathrm{A})$ modification in the regulation of circRNAs. MOL CANCER 19:105. doi:10.1186/s12943-02001224-3

22. Chen $Y$, Lin $Y$, Shu Y, He J, Gao W (2020) Interaction between $N(6)$-methyladenosine (m(6)A) modification and noncoding RNAs in cancer. MOL CANCER 19:94. doi:10.1186/s12943-020-01207-4

23. Zheng ZQ, Li ZX, Zhou GQ, Lin L, Zhang LL, Lv JW, Huang XD, Liu RQ, Chen F, He XJ, Kou J, Zhang J, Wen X, Li YQ, Ma J, Liu N, Sun Y (2019) Long Noncoding RNA FAM225A Promotes Nasopharyngeal Carcinoma Tumorigenesis and Metastasis by Acting as ceRNA to Sponge miR-590-3p/miR-1275 and Upregulate ITGB3. CANCER RES 79:4612-4626. doi:10.1158/0008-5472.CAN-19-0799

24. Chen RX, Chen X, Xia LP, Zhang JX, Pan ZZ, Ma XD, Han K, Chen JW, Judde JG, Deas O, Wang F, Ma NF, Guan X, Yun JP, Wang FW, Xu RH, Dan X (2019) N(6)-methyladenosine modification of circNSUN2 facilitates cytoplasmic export and stabilizes HMGA2 to promote colorectal liver metastasis. NAT COMMUN 10:4695. doi:10.1038/s41467-019-12651-2

25. Wu Y, Yang X, Chen Z, Tian L, Jiang G, Chen F, Li J, An P, Lu L, Luo N, Du J, Shan H, Liu H, Wang H (2019) m(6)A-induced IncRNA RP11 triggers the dissemination of colorectal cancer cells via upregulation of Zeb1. MOL CANCER 18:87. doi:10.1186/s12943-019-1014-2

26. Wu G, Xue M, Zhao Y, Han Y, Li C, Zhang S, Zhang J, Xu J (2020) Long noncoding RNA ZEB1-AS1 acts as a Sponge of miR-141-3p to Inhibit Cell Proliferation in Colorectal Cancer. INT J MED SCI 17:1589-1597. doi:10.7150/ijms.46698

27. Jin Z, Chen B (2020) LncRNA ZEB1-AS1 Regulates Colorectal Cancer Cells by MiR-205/YAP1 Axis. Open Med (Wars) 15:175-184. doi:10.1515/med-2020-0026

28. Ni X, Ding Y, Yuan H, Shao J, Yan Y, Guo R, Luan W, Xu M (2020) Long non-coding RNA ZEB1-AS1 promotes colon adenocarcinoma malignant progression via miR-455-3p/PAK2 axis. Cell Prolif 53:e12723. doi:10.1111/cpr.12723

29. Lv SY, Shan TD, Pan XT, Tian ZB, Liu XS, Liu FG, Sun XG, Xue HG, Li XH, Han Y, Sun LJ, Chen L, Zhang LY (2018) The IncRNA ZEB1-AS1 sponges miR-181a-5p to promote colorectal cancer cell proliferation by regulating Wnt/ $\beta$-catenin signaling. CELL CYCLE 17:1245-1254.

doi:10.1080/15384101.2018.1471317

30. Xiong WC, Han N, Wu N, Zhao KL, Han C, Wang HX, Ping GF, Zheng PF, Feng H, Qin L, He P (2018) Interplay between long noncoding RNA ZEB1-AS1 and miR-101/ZEB1 axis regulates proliferation and 
migration of colorectal cancer cells. AM J TRANSL RES 10:605-617

31. Shen Y, Gao X, Tan W, Xu T. STAT1-mediated upregulation of IncRNA LINC00174 functions a ceRNA for miR-1910-3p to facilitate colorectal carcinoma progression through regulation of TAZ. GENE. (2018)666: 64-71. doi:10.1016/j.gene.2018.05.001

32. Malki A, EIRuz RA, Gupta I, Allouch A, Vranic S, Al MA. Molecular Mechanisms of Colon Cancer Progression and Metastasis: Recent Insights and Advancements. INT J MOL SCI.(2020)22. doi:10.3390/ijms22010130

33. Koveitypour Z, Panahi F, Vakilian M, Peymani M, Seyed FF, Nasr EM, Ghaedi K (2019) Signaling pathways involved in colorectal cancer progression. CELL BIOSCI 9:97. doi:10.1186/s13578-0190361-4

\section{Figures}




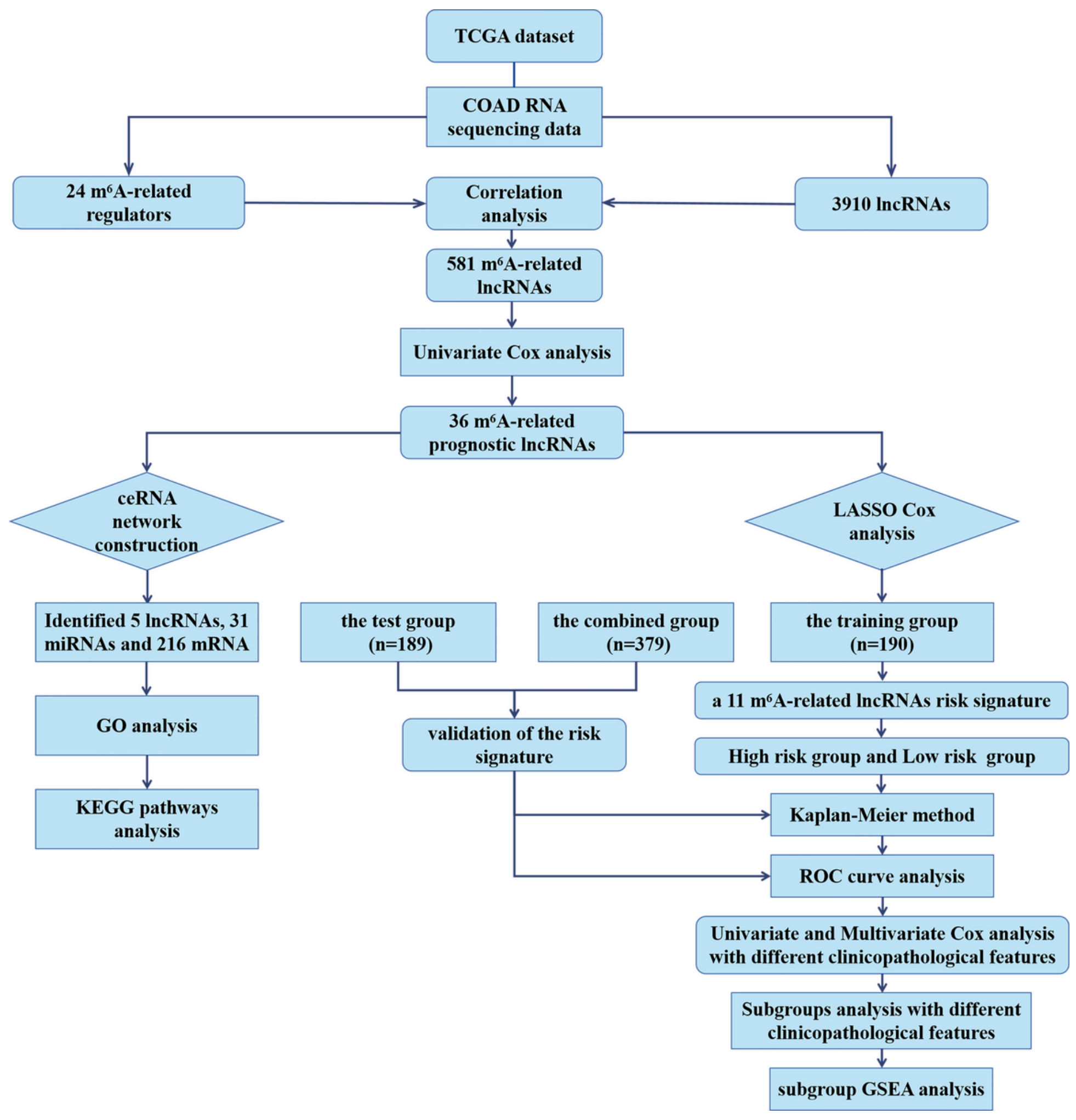

Figure 1

The flow chart of the study design and analysis. 
A

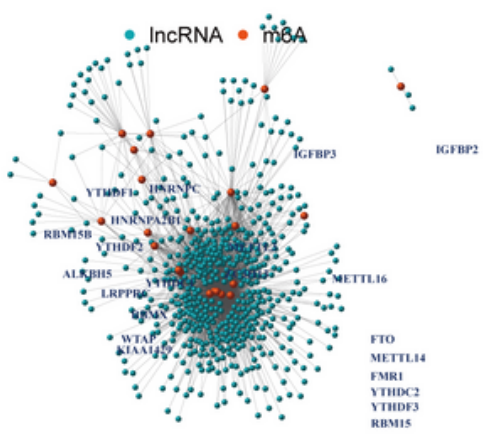

B

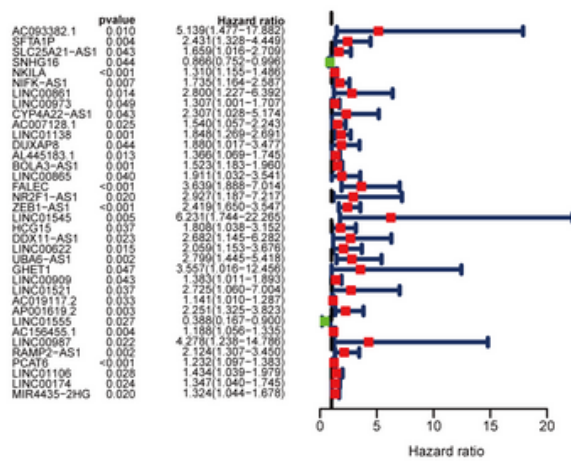

$\mathrm{C}$

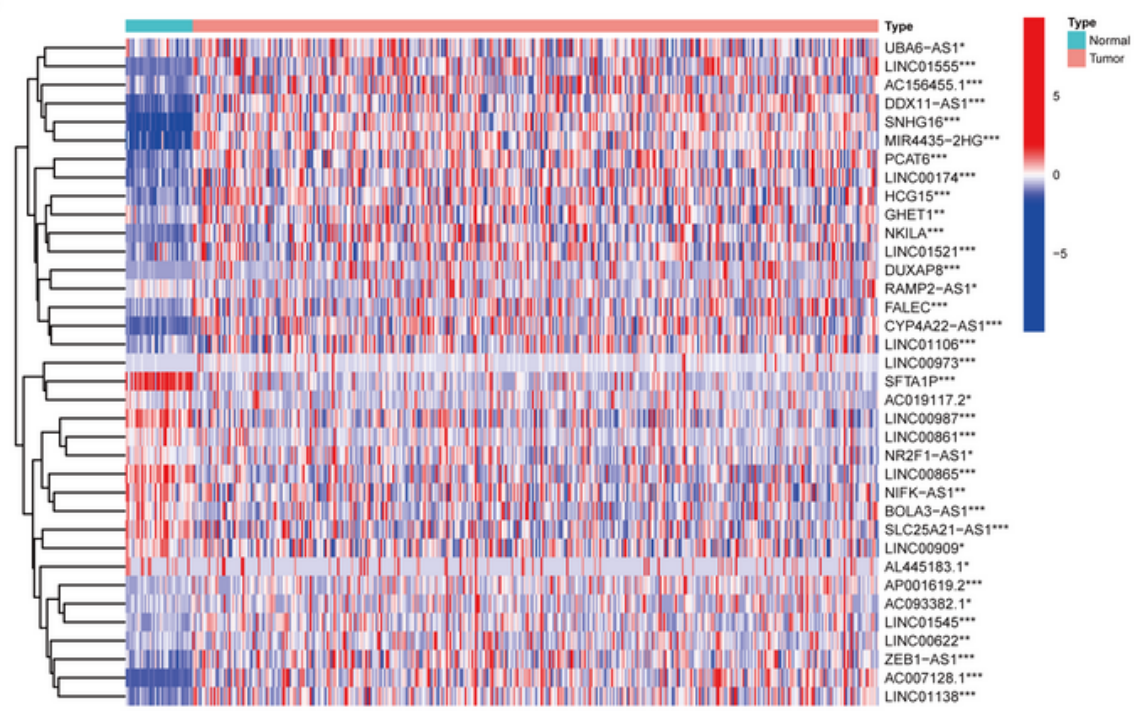

D

Type 追 Normal 追 Tumor

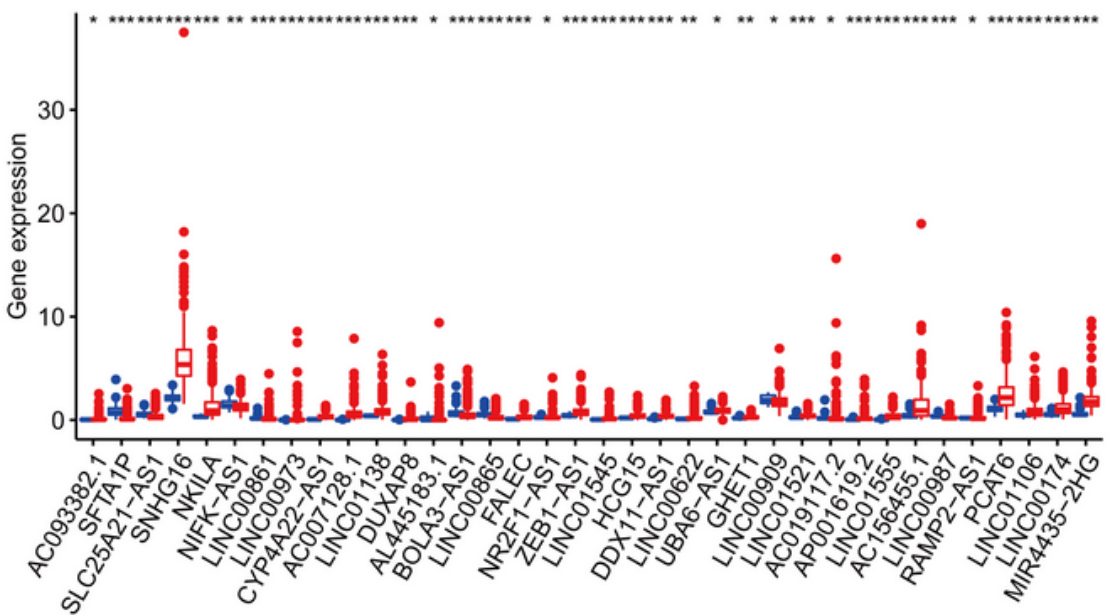

Figure 2

Identification of m6A-related prognostic IncRNAs in COAD patients. (A) The network of the $24 \mathrm{~m} 6 \mathrm{~A}$ related regulators and 581 m6A-related IncRNAs. (B) The Hazard ratio (HR) 95\% confidence interval (Cl) of 36 m6A-related IncRNAs estimated by univariate Cox regression. (C, D) The expression of 36 prognostic m6A-related IncRNAs in TCGA database between the tumor group and the normal group. 
A

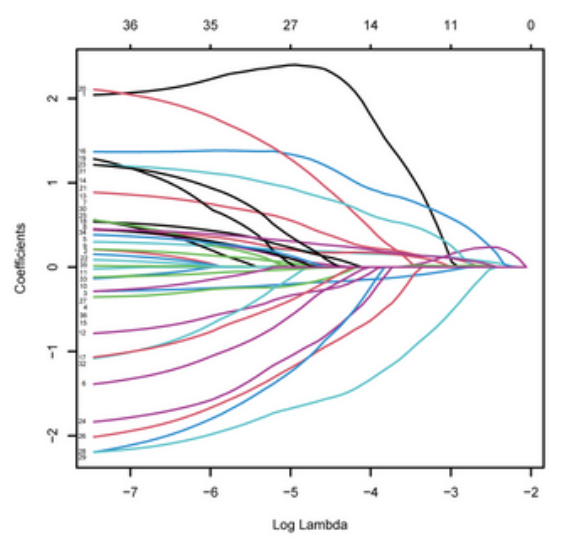

B

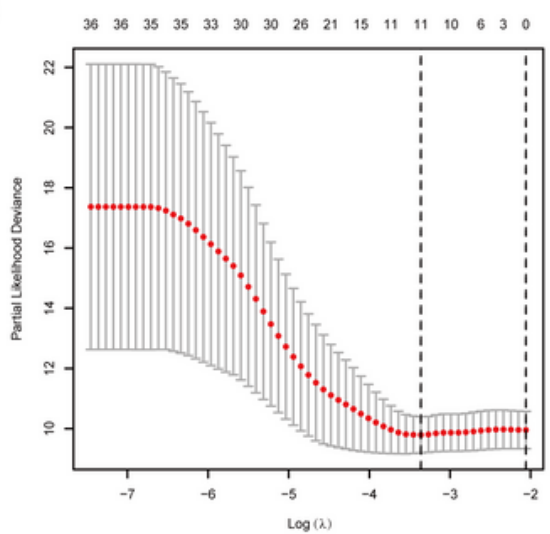

C
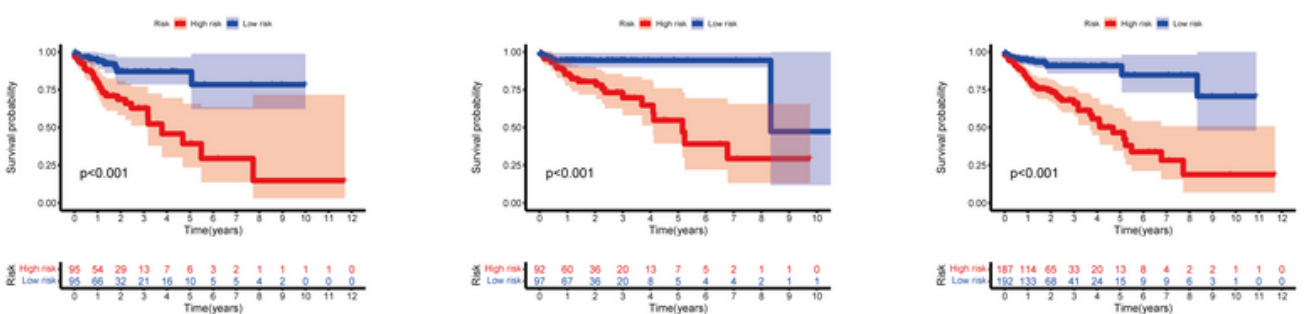

D
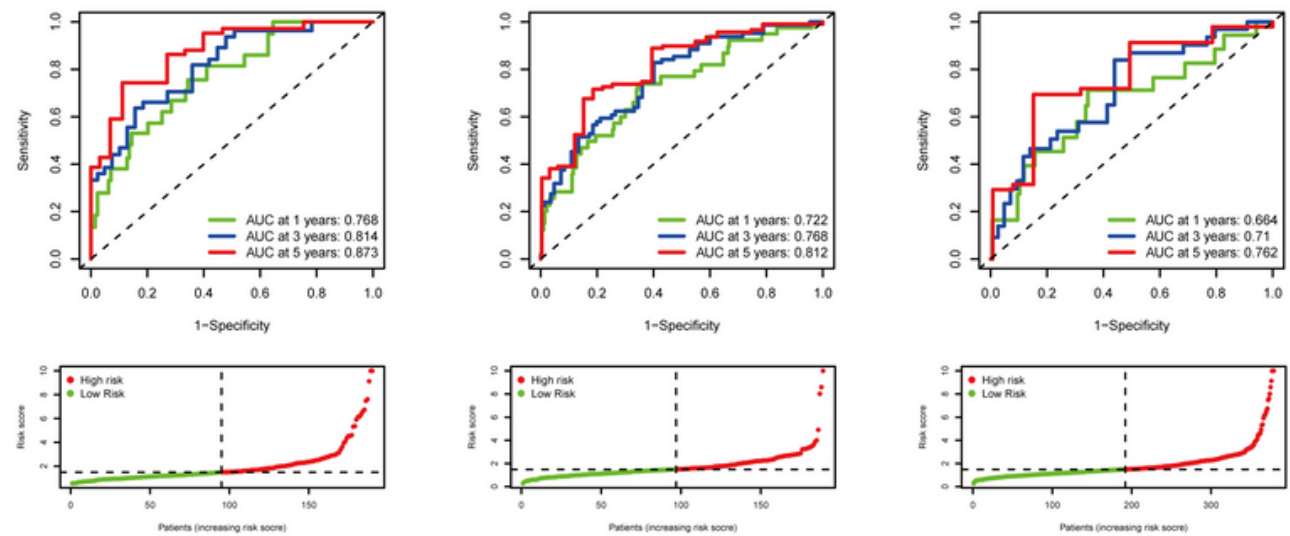

F
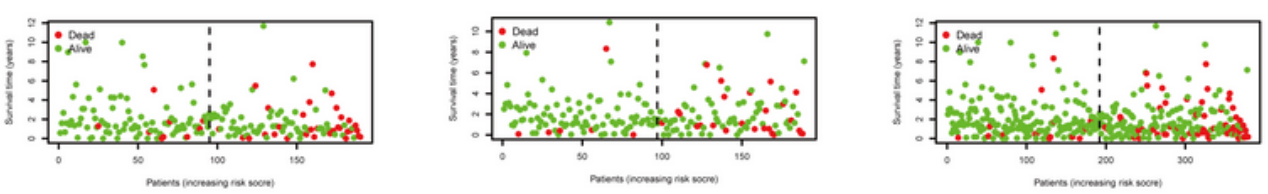

G
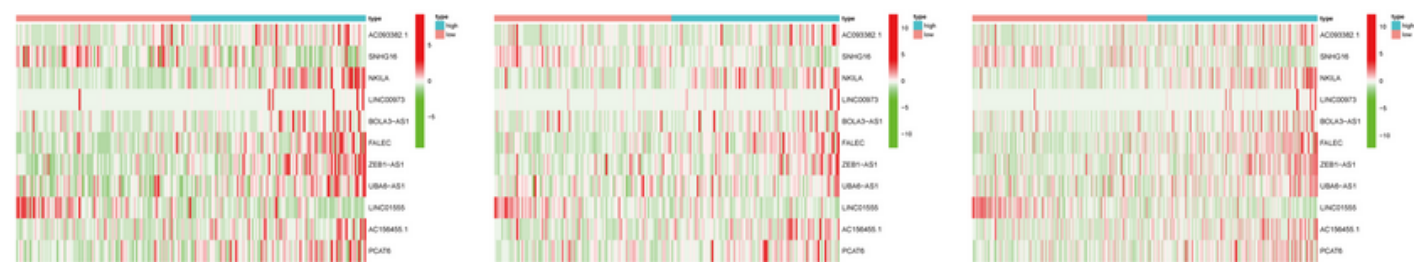

Figure 3

Construction and Verification of the m6A-Related IncRNAs Prognostic Signature. (A) The point with the smallest cross verification error corresponds to the number of factors included in the LASSO regression model. (B) The lines of different colors represent the trajectory of the correlation coefficient of different factors in the model with the increase of Log Lamda. (C) Kaplan-Meier analysis of patients in the highrisk group and low-risk group in the training group, the test group and the combined group. (D) ROC 
analysis of 1-3- and 5-year in the training group, the test group and the combined group. (E) Distribution of patients with different risk scores in the training group, the test group and the combined group. (F) OS status of patients with different risk scores in the training group, the test group and the combined group. (G) Heatmap of the prognostic signature scores in the training group, the test group and the combined group.

A

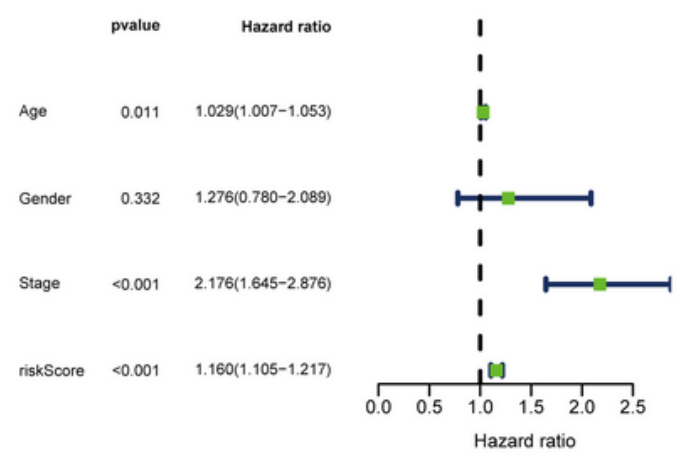

B

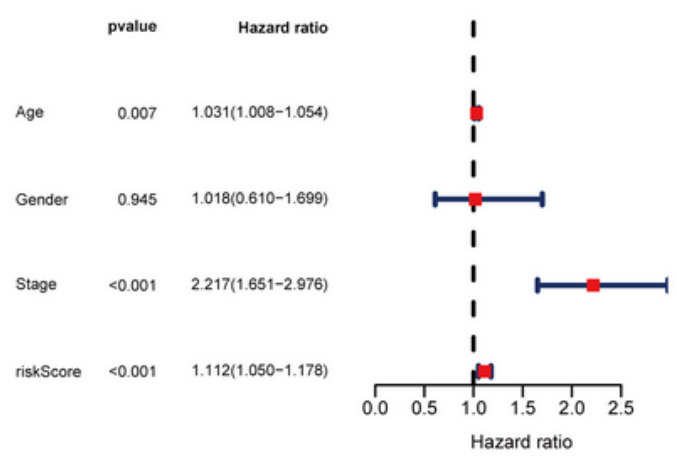

$\mathrm{C}$

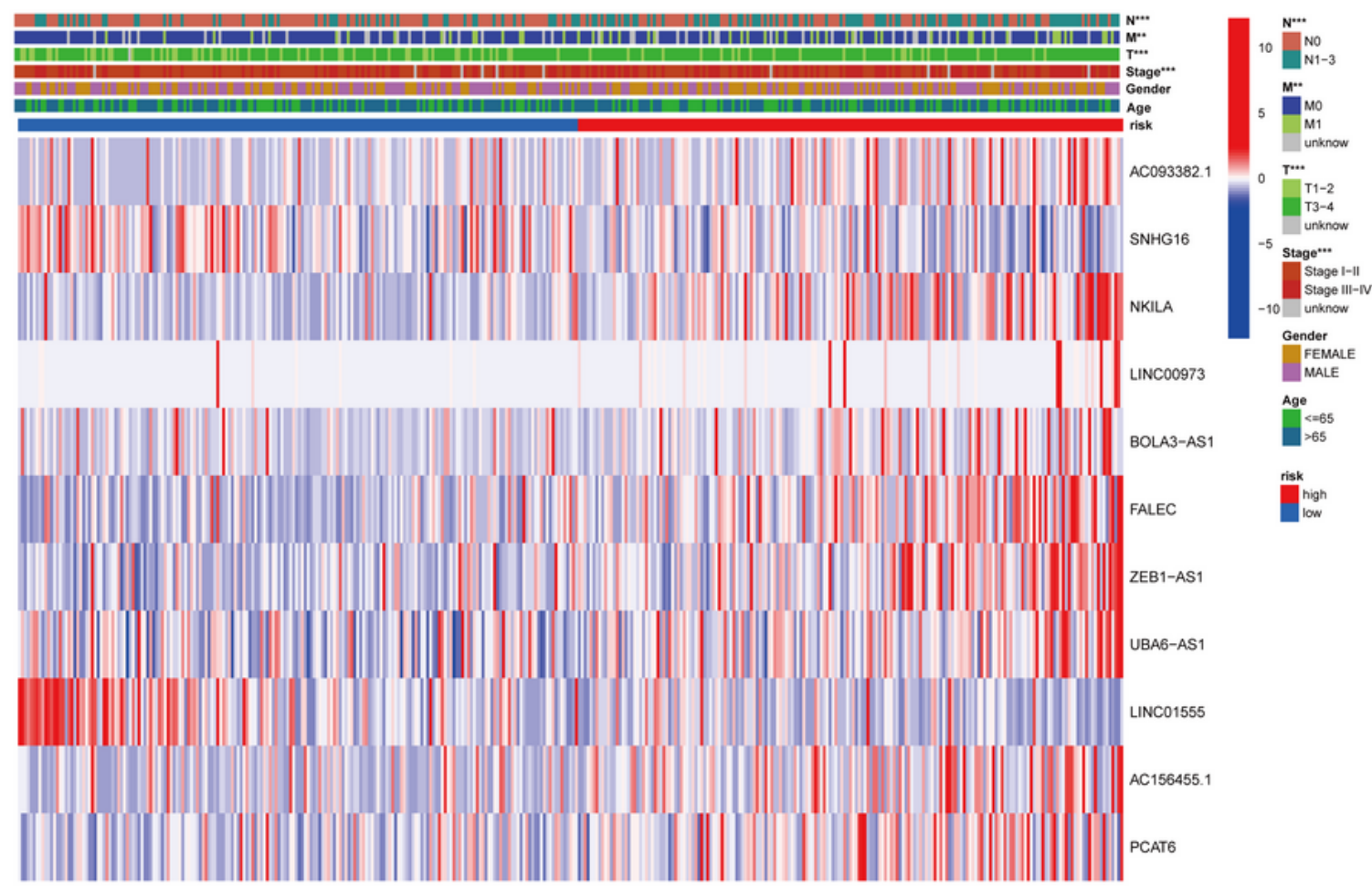

$\mathrm{D}$
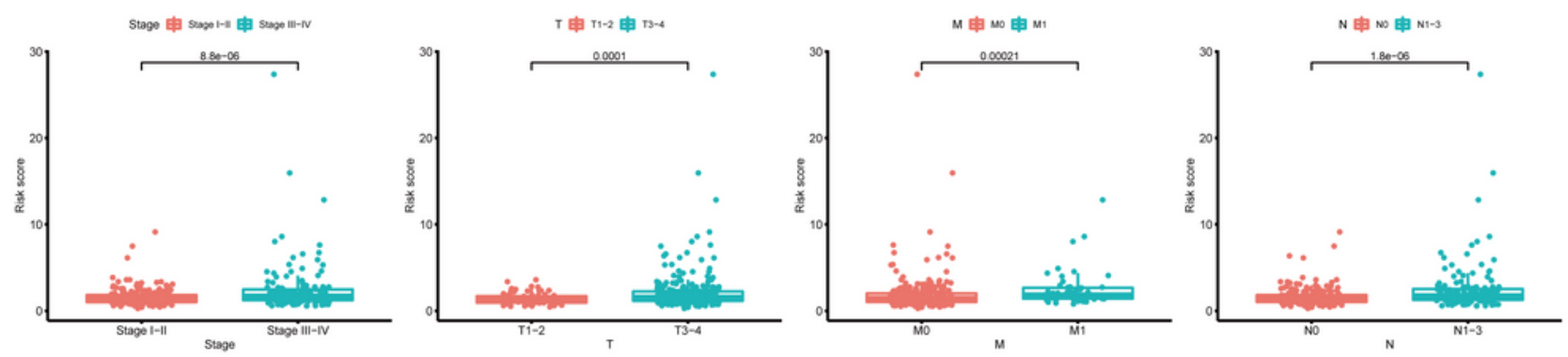

Figure 4 
Relationship between the risk score and clinicopathological characteristics. (A) Univariate Cox regression analysis of the association between clinicopathological factors (including risk score) and overall survival of patients in the training group, the test group and the combined group. (B) Multivariate Cox regression analysis of the association between clinicopathological factors (including risk score) and overall survival of patients in the training group, the test group and the combined group. (C) The heatmap showed the clinicopathological characteristics in high-risk group and low-risk group. The distribution of clinicopathological characteristics was compared between the high-risk group and low-risk groups. * $p$ $<0.05, * \star p<0.01, * \star \star p<0.001$. (D) Pathological stage, T stage, $M$ stage and $N$ stage were significantly different in high-risk and low-risk group. 
A

Patients with age $<=65$

Risk = high = low
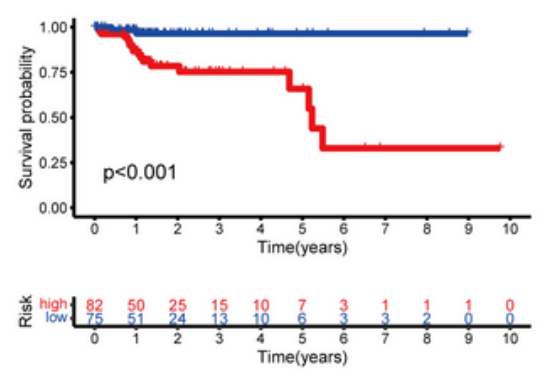

D

Patients with MALE

Risk $=$ high $=10 \mathrm{w}$
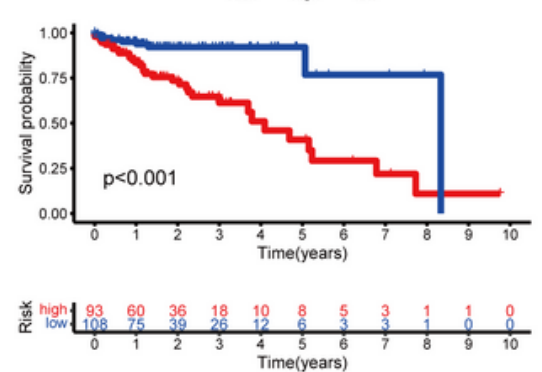

G

Patients with T1-2
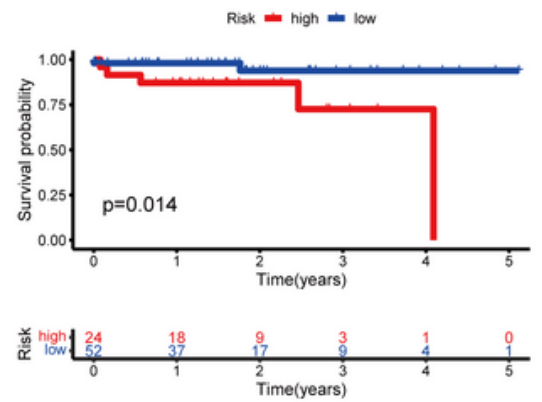

J

Patients with NO

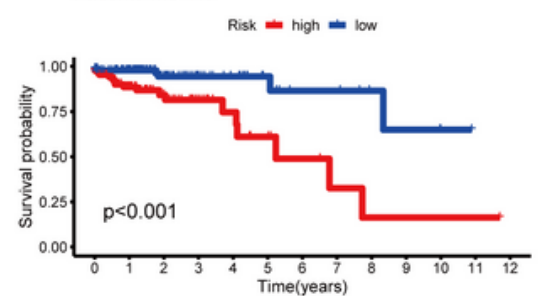

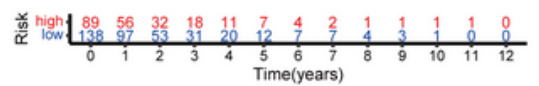

B

Patients with age $>65$

Risk $=$ high $=10 \mathrm{w}$

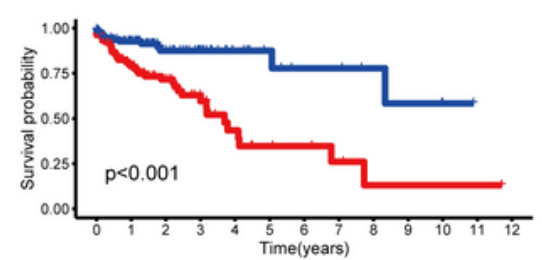

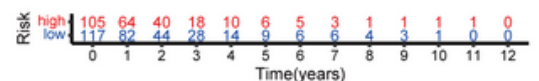

E

Patients with Stage I-II

Risk = high = low

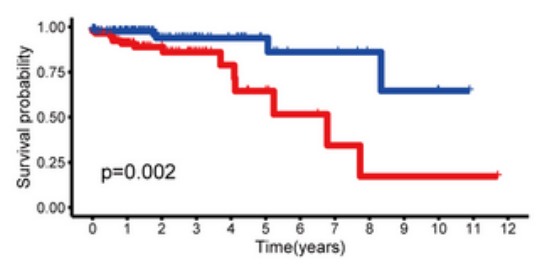

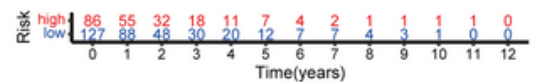

$\mathrm{H}$

Patients with T3-4

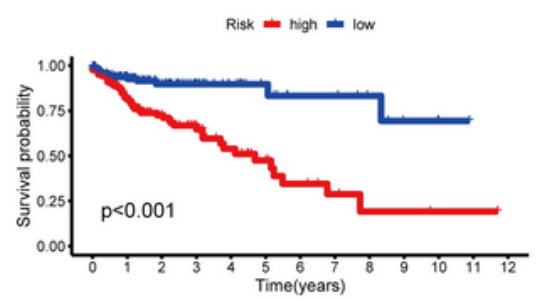

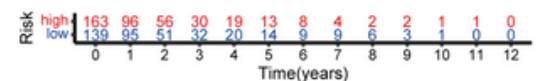

K

Patients with $\mathrm{N} 1-3$

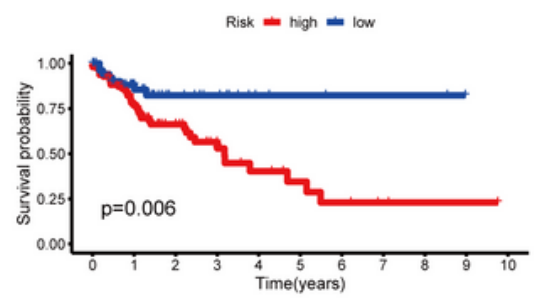

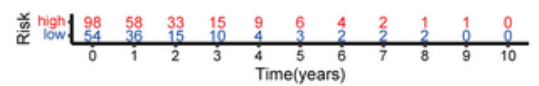

C

Patients with FEMALE

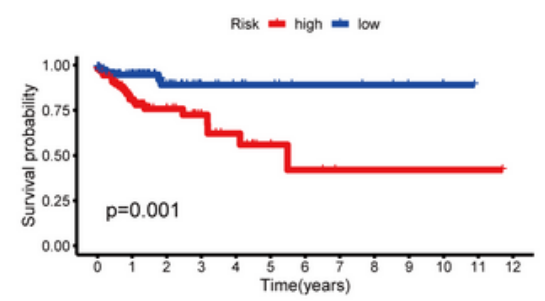

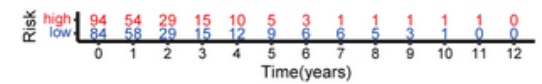

F

Patients with Stage III-IV

Risk $=$ high $=10 \mathrm{w}$

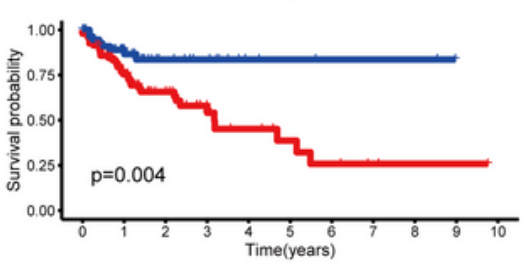

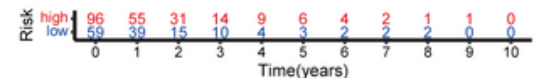

I

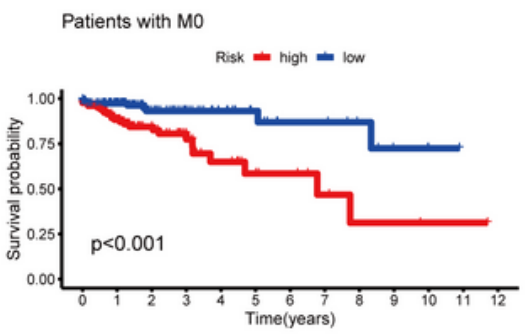

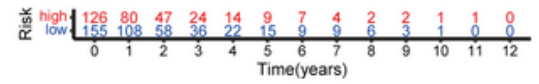

\section{Figure 5}

Subgroup Analysis with Different Clinicopathological Features in COAD. (A)Age $\leq 65$. (B)Age $>65$.

(C)Female. (D)Male. (E)stage I-II. (F)stage III-IV. (G)T1-2 stage. (H)T3-4 stage. (I)MO stage. (J)NO stage.

(K)N1-3 stage. 
A

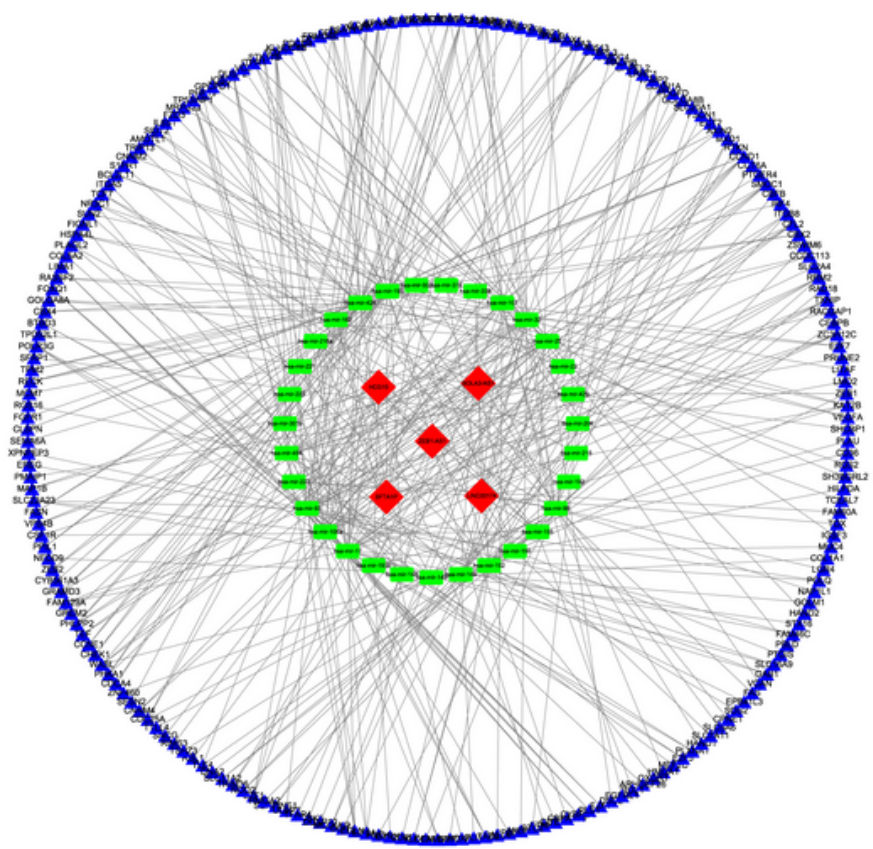

B

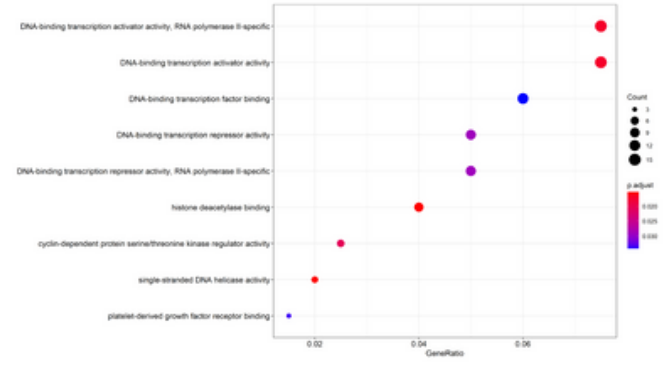

C

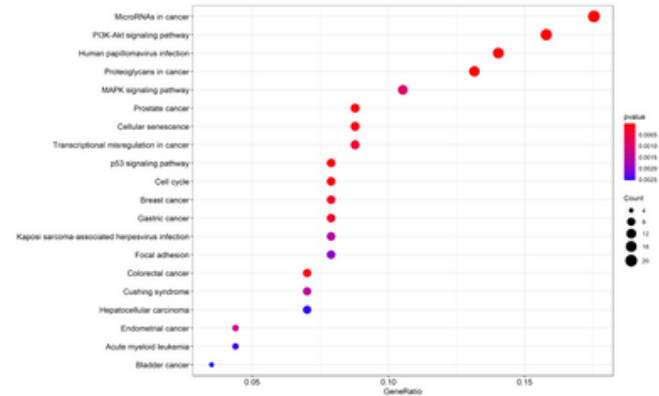

D
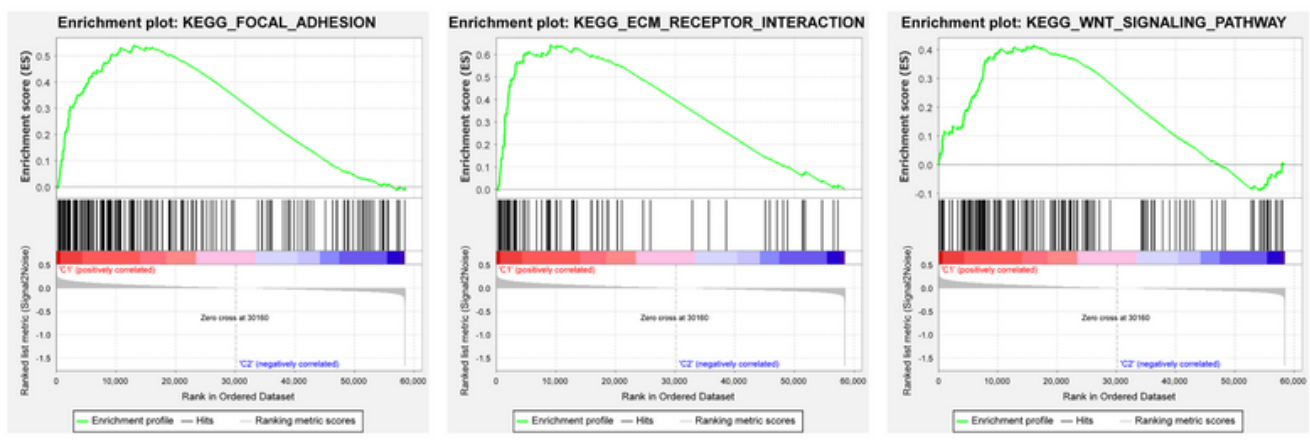

\section{Figure 6}

The ceRNA network construction and biological function analysis. (A)The ceRNA network of 5 IncRNAs (red) and their target miRNAs (green) and mRNAs (blue).(B) GO analysis in 216 target mRNAs. (C) KEGG pathways analysis in 216 target mRNAs. (D) The high-risk groupwas associated with "Focal adhesion," "ECM-receptor interaction," and "Wnt signaling pathway." 